\title{
Topographic Influence on the Pattern of Flow through Bass Strait
}

\author{
Peter G. Baines and David L. Murray \\ CSIRO Division of Atmospheric Research, Aspendale, Vic. 3195, Australia.
}

\begin{abstract}
A laboratory simulation of the flow through Bass Strait has been made with the aid of a physical model of the topography, situated in a tank on a rotating turntable. The throughflow was driven by a continuous supply of homogeneous water to the western end that was uniform with latitude. The effect of the depth variations within the strait were realistically represented and had a controlling influence on the flow pattern. In particular, the uniform inflow from the west was directed towards the south-eastern region, guided along contours of constant depth around the central depression by approximate conservation of potential vorticity. West of Flinders Island, the flow then splits into a northward branch that flows toward the Victorian coast and leaves the strait near Gabo Island and a southern branch that leaves through Banks Strait. This modelled flow pattern is generally consistent with the outflows inferred from observed temperature and salinity fields.
\end{abstract}

\section{Introduction}

In winter the mean flow of water through Bass Strait is from west to east and occurs in surges lasting for about three days, with fluxes of up to 3 Sverdrups $\left[3 \times 10^{6} \mathrm{~m}^{3} \mathrm{~s}^{-1}\right]$ or more (Baines et al. 1991). These surges are driven by the local wind stress and the wind stress over the continental shelf further west, with the latter being generally more important by a factor of about two. At the eastern escarpment the Bass Strait water is denser than the upper Tasman Sea water, and the former flows down the continental slope to a depth of about $400 \mathrm{~m}$.

Observations of this 'underwater waterfall' phenomenon are few and are largely restricted to those reported by Tomczak (1985), who measured the cascade during the winter of 1981. Fig. 1 shows the paths of this flow at the eastern end of the strait as inferred by Tomczak (1985) from his observations. In this figure, most of the overflow occurs at the north-eastern corner of the strait. However, some fluid is depicted as exiting from Bass Strait through Banks Strait (at the south-eastern corner, between Cape Barren Island and Tasmania) and then turning north to join the remainder in the overflow. The reason for this behaviour was not clear from the observations. The laboratory experiment described here was carried out to investigate the pattern of this throughflow within eastern Bass Strait in general, including the possible flow through Banks Strait.

The location and character of the overflow have been explored with two laboratory experiments: a large-scale one to study the overall flow pattern, and a small-scale one to study the local dynamics. The results of the latter are described elsewhere (Mitsudera and Baines 1992). This paper is concerned with the large-scale experiment, which involves a rotating three-dimensional scale model of the eastern end of Bass Strait. The object of the experiment is to explore the factors affecting the location and behaviour of the overflow, to provide a description of it as seen in the model, and to assess the relevance of this type of modelling to continental shelf and coastal flows in general.

The throughflow within Bass Strait is largely governed by the linearized shallow-water equations with turbulent bottom friction (Baines et al. 1991). Scale analysis shows that the time-dependent and bottom friction terms are relatively small so that the flow is approximately geostrophic, and that the horizontal scales are large so that $|\zeta| f \mid \ll 1$, where $\zeta$ is the relative vorticity and $f$ is the Coriolis parameter. The experiment should reproduce these balances, and the primary interest is in the effect of geometry and bottom variations.

\section{The Experiment}

The geometry and depth contours of the central and eastern part of Bass Strait are shown in Fig. 2. The scale model of Bass Strait (see Fig. 3a) was constructed from polystyrene and plastic and was accommodated within a circular tank of diameter $110 \mathrm{~cm}$. The Flinders and Cape Barren Islands group was represented as a single unit. The scale in the vertical was stretched by a factor of $200: 1$, and variations in the depth within the strait shown in Fig. 2 were reproduced. The tank was filled to a depth of $25 \mathrm{~cm}$ with either homogeneous or stratified water, giving a typical depth within Bass Strait of $3.0 \mathrm{~cm}$. The whole tank was placed on a rotating turntable and, for a given experiment, 


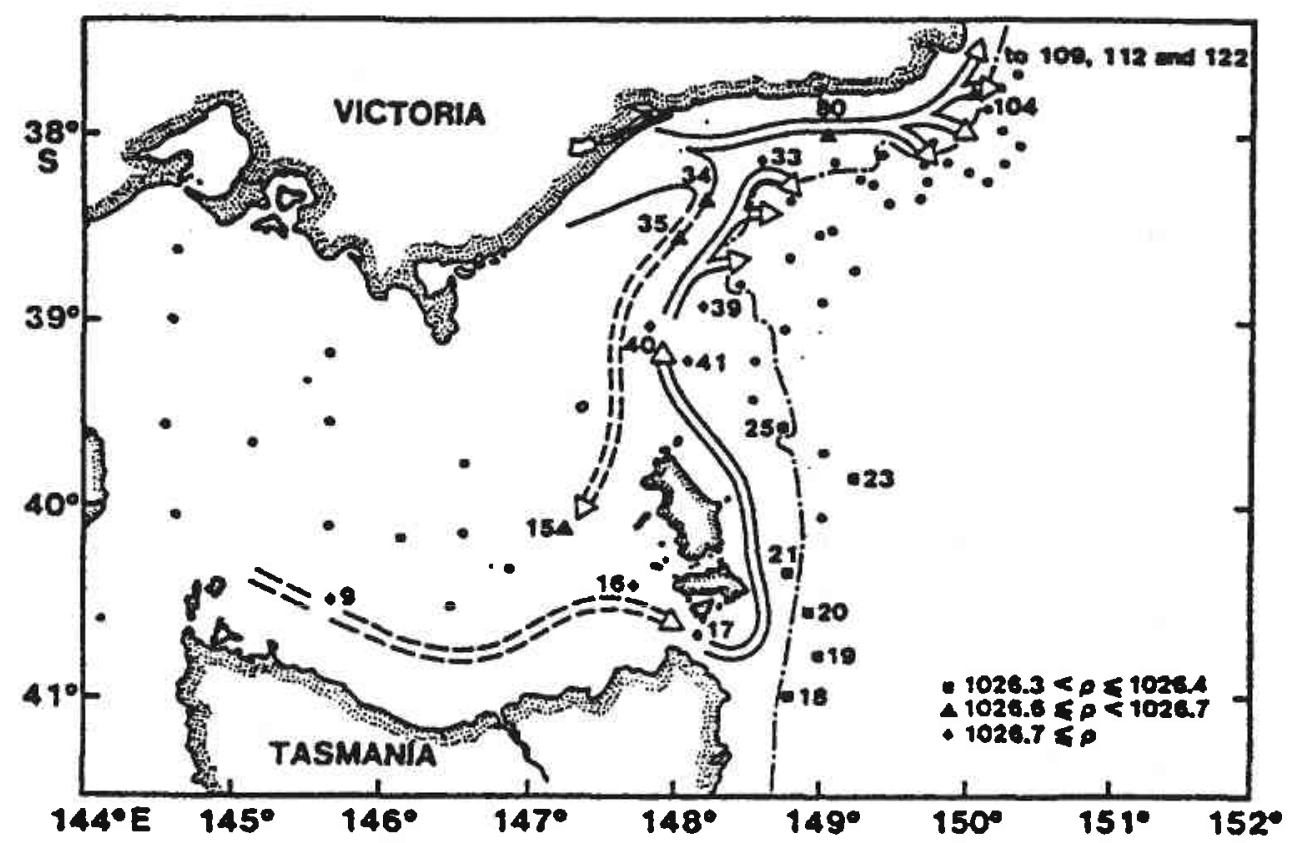

Fig. 1. Possible paths of the water before cascading over the slope in winter 1981, inferred by Tomczak (1985). Dots denote the coarse grid of stations used but do not fully represent more closely spaced stations used on a finer scale in the north-east. The dashed lines are more conjectural than the solid lines.

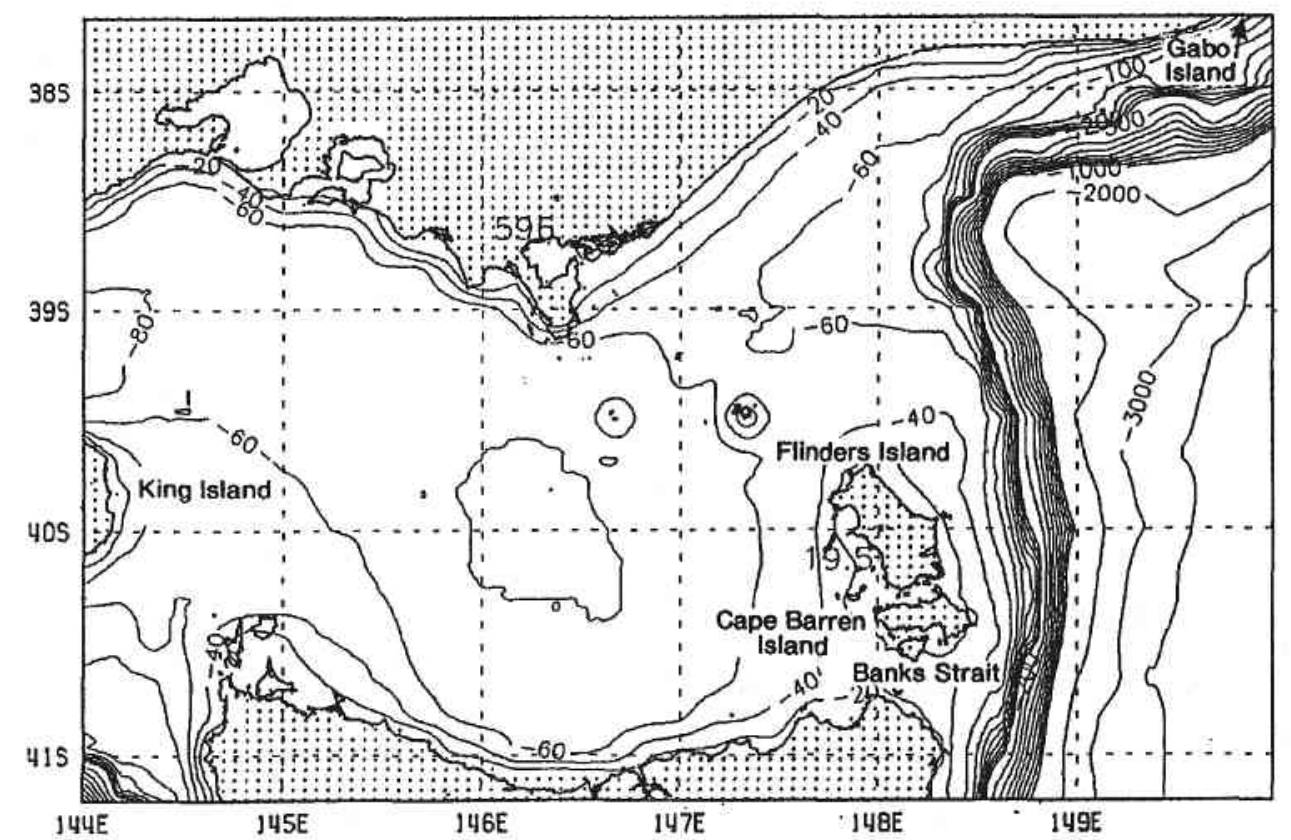

Fig. 2. Topography and bathymetry of the central and eastern portion of Bass Strait. Depth contours are in metres, with the central depths over $80 \mathrm{~m}$. (Courtesy of G. D. Hubbert, Australian Bureau of Meteorology.)

was spun up to an approximate state of rigid rotation. It transpired that this was a non-trivial task because the inequality in depths tended to magnify the effects of oscillations in the fluid motion that appeared at the rotational frequency. These oscillations were negligible in the deep water but were significant in the shallow water, 
where they complicated measurement of the mean flow, and conditions were chosen to minimize them as described below.

Field observations (Baines et al. 1991) have shown that in winter the water in the western region of Bass Strait is not stratified and the throughflow is approximately uniform with latitude north and south of King Island (with a slight increase at the southern end). Hence it is appropriate to represent this flow by an imposed uniform eastwards velocity in the western part of the strait. This inflow from the west was realized by releasing salty water at a uniform rate into a reservoir behind a diffuser (made from coarse matting) that was aligned in the north-south direction and located at $145^{\circ} \mathrm{E}$ (approximately), and the resulting flux was approximately uniform with latitude.

Two techniques of flow visualization were employed. Firstly, the inflowing fluid was dyed, and secondly, in other experiments small polystyrene beads were introduced into the inflowing fluid and used as tracers, their positions being recorded on tape by a video camera viewing from above. It was found that it was not adequate to simply place these beads on the surface because the velocity there was significantly less than the mean, apparently because of the effect of surface tension (this effect is not thought to be important for these flows overall, but it deserves further study). To circumvent this problem, the fluid was illuminated by a horizontal sheet of laser light at a depth of approximately $1 \mathrm{~cm}$ below the surface, and the velocities of the beads in this beam were recorded. This velocity field was consistent with the overall flux.

The parameters in these experiments were the rotation frequence $\Omega$, the total flow rate $Q$, the mean depth $\bar{D}$ of the water in the strait, the difference in density $\left(\Delta \rho=\rho_{\mathrm{i}}-\rho_{0}\right)$ between that of the inflowing fluid $\rho_{\mathrm{i}}$ and that of the ambient fluid at the surface $\rho_{0}$ the kinematic viscosity $v$ and (of peripheral relevance here) the buoyancy frequency of the deep water $N$. Since the flow pattern within Bass Strait is primarily barotropic, it is essential that the barotropic Rossby radius of deformation $\lambda \equiv(g \bar{D})^{1 / 2} / 2 \Omega$ have the same value relative to the horizontal scale of the model as it does in the ocean. Given this, the flow pattern within the strait was not sensitive to the values of $Q$ and $\bar{D}$ provided that these were large enough so that friction was not dominant and $Q$ was not so large that advective terms were important (or the vorticity was comparable with $f$ ). For Bass Strait, with $\bar{D}=70 \mathrm{~m}$ and the Coriolis parameter $f=9.0 \times 10^{-5} \mathrm{rad}$ $\mathrm{s}^{-1}, \lambda \equiv(g \bar{D})^{1 / 2} / f=290 \mathrm{~km}$. To give the same ratio to the horizontal scale in the model then requires $\lambda=36.5 \mathrm{~cm}$. This may be achieved by taking $\bar{D}=3.0 \mathrm{~cm}$ and rotation period $2 \pi / \Omega=8.5 \mathrm{~s}$ (other values close to this were sometimes used). This is the largest value of $\bar{D}$ that is practicable with this model, and it also minimizes the oscillations mentioned above.
$Q$ was varied in the range $1-3 \mathrm{~L} \mathrm{~min}-1$. Typical fluid velocities $U$ in the strait were less than $0.5 \mathrm{~cm} \mathrm{~s}^{-1}$ with horizontal scales of motion $L \simeq 5 \mathrm{~cm}$, so that Rossby number $R_{0}=U L / 2 \Omega<0.07 \ll 1$. The motion was observed to be laminar, with a bottom Ekman layer of thickness $(v / \Omega)^{1 / 2} \simeq 0.1 \mathrm{~cm}$. The ratio of viscous bottom stress to Coriolis force for the whole fluid layer is then $O\left[(v / \Omega)^{1 / 2} / \bar{D}\right]$ which is very small $(\sim 0.04)$. Hence the motion is mostly geostrophic, except in the downstream direction near boundaries. Within the strait the vorticity equation for the vertically integrated motion is

$$
\frac{\mathrm{D}}{\mathrm{D} t}\left(\frac{\zeta+2 \Omega}{D}\right)=\nabla \times \tau
$$

where $D$ is local depth, $\zeta$ is the relative vorticity, and $\tau$ is the viscous stress on the fluid due to the rigid bottom. In the model, where a depth $\bar{D}$ of $3 \mathrm{~cm}$ corresponds to $70 \mathrm{~m}$ in the ocean, depths of 2.5 and $3.5 \mathrm{~cm}$ correspond to 60 and $80 \mathrm{~m}$ respectively, which covers the range of interest. These figures give $\Delta D \bar{D}=1 / 3$ in the model, which corresponds to $2 / 7$ in the ocean, so that the corresponding changes in potential vorticity scale appropriately.

Baroclinic effects due to the density difference were generally negligible in the strait because it mostly became filled with inflowing water, but they could be significant near the shelf break, where a relatively stationary boundary may develop. If these effects are to be realistically modelled in the experiment, the baroclinic Rossby deformation radius $\left(g^{\prime} D\right)^{1 / 2} / 2 \Omega$ ) (where $\left.g^{\prime}=g \Delta \rho / \rho_{0}\right)$ must also have the correct value, which implies that $\Delta \rho / \rho_{0}$ must have the same value in the laboratory as in the ocean. From the data of Tomczak (1985) this difference is very small $\left(\Delta \rho / \rho_{0} \simeq 0.0004\right)$. This implies much smaller scales of motion than the barotropic, which must be correctly related to the local topographic scales. The experiments performed have covered a range of values from zero to 0.026 , but because of the difficulties in representing these small scales they do not model the baroclinic processes at the shelf break in a realistic way. However, the main focus of this paper is the barotropic flow pattern within the strait, and this was found to be insensitive to $\Delta \rho / \rho_{0}$.

\section{Results}

Each experimental run was begun by commencing the inflow of fluid to the reservoir and simultaneously lifting the barrier between the reservoir and the region of interest. In the case of dyed fluid, the spread of the dye showed the development of the pattern of motion, and the flow approached an approximately steady state when the leading edge of the released fluid passed out of the strait. Several runs with different values of $\Delta \rho / \rho_{0}$ ranging from 0.026 to zero were made, and in each case the flow pattern within Bass Strait was qualitatively the same. Fig. 3 shows the 
(a)

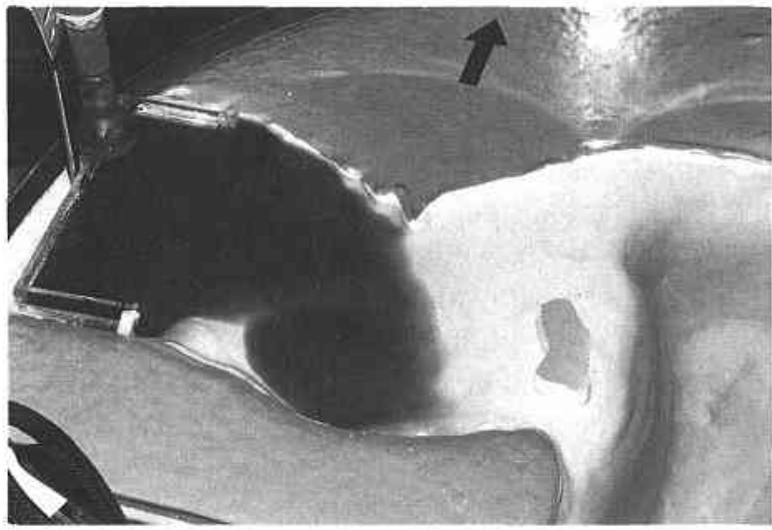

(b)

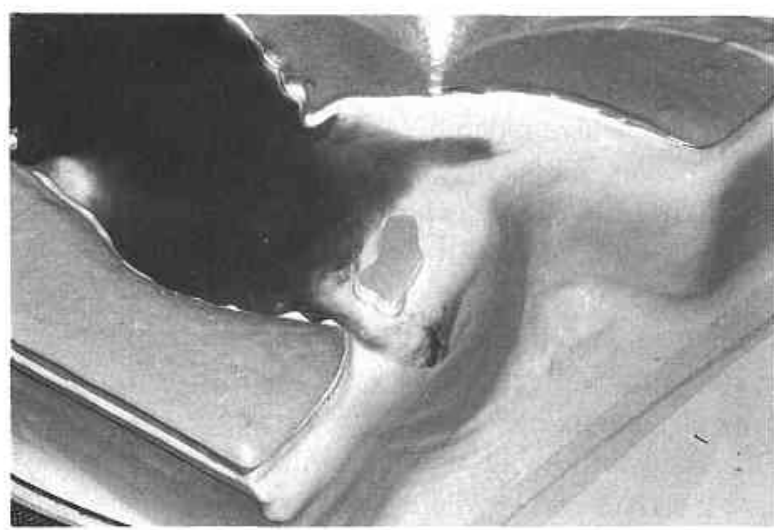

(c)

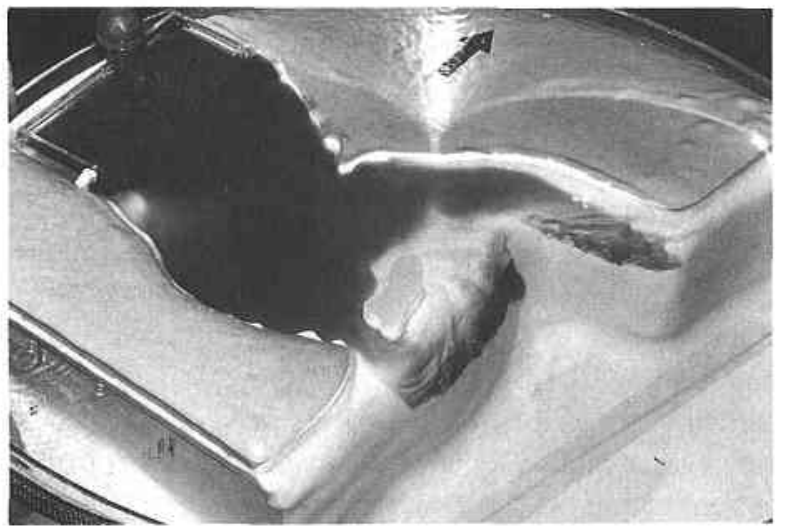

Fig. 3. Typical experiment with released dye, showing the dye pattern at three different times. Here, $Q=3 \mathrm{~L} \mathrm{~min}^{-1}, \bar{D}=3.0 \mathrm{~cm}, \Delta \rho / \rho_{0}=0.026$, rotation period $2 \pi / \Omega=6.7$, and $N=1.5 \mathrm{rad} \mathrm{s}^{-1}$. The arrow denotes north. The tendency of the inflowing dyed fluid to flow clockwise around the depth contours of Fig. 2 is shown in (a).

pattern of dye at three different times for a typical run with $\Delta \rho / \rho_{0}=0.026$. This shows that the fluid tends to flow toward the south-eastern corner, where (in Figs $3 b$ and $3 c$ ) it splits, with some fluid flowing through Banks Strait to the south and most flowing north to the north-eastern corner. In

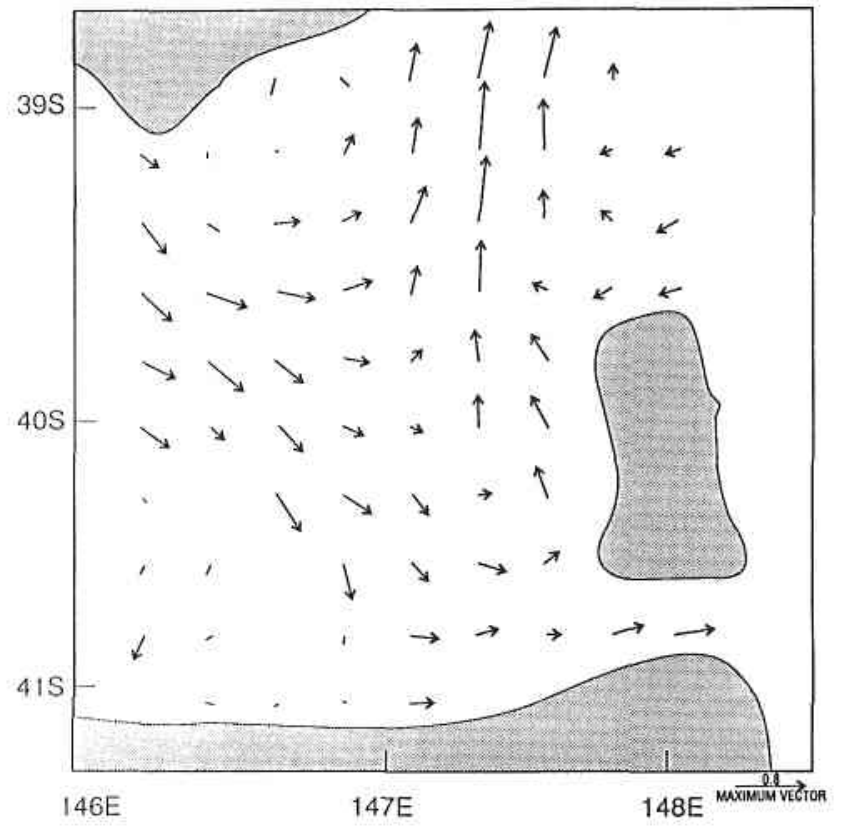

Fig. 4. Velocity field from an experiment with $Q=1 \mathrm{~L} \mathrm{~min}^{-1}, \rho_{0}=0, \bar{D}$ $=3.0 \mathrm{~cm}$, rotation period $9.3 \mathrm{~s}$, and $N=0$, measured by video recording of tracers. The longest vector denotes $0.8 \mathrm{~cm} \mathrm{~s}^{-1}$.

this example, the density difference is so large that downflow over the continental slope occurs quite close to Banks Strait, and this deeper fluid then flows north. This northward flow in or over the deeper water occurred regardless of the value of the density difference. For runs with smaller density differences this downslope flow occurred progressively further north, but no significant effect of varying this density difference on the flow pattern within Bass Strait could be discerned.

With inflowing fresh water $(\Delta \rho=0)$ the flow through Banks Strait was still strong, with northward flow over the shelf to the east of the Flinders Island group. Fig. 4 shows a velocity field for freshwater inflow obtained with the video data acquisition system and tracer beads. For this case the flow through the two gaps north and south of Flinders Island was summed by numerical integration, and the flow through Banks Strait was observed to be $20 \%$ of the total flux through Bass Strait, with the Banks Strait flow rejoining the northern branch as shown. Hence the experiment reproduces the phenomenon of a significant flux of fluid leaving Bass Strait through Banks Strait, but with most of the Bass Strait flow following a more direct path well north of Flinders Island and leaving in the north-eastern corner, as inferred by Tomczak (1985). It remains to be explained why this is the case.

Eqn 1 shows that if $\zeta$ and $\nabla \times \tau$ are negligible, the fluid flows along lines of constant depth $D$. This is not strictly 
possible in the flows studied here, but Figs $3 a$ and $3 b$ show that the fluid does have a strong tendency to follow the depth contours, flowing northward and around the central depression. The net effect is to guide the fluid to the southeastern corner of Bass Strait, where the resulting increase in pressure causes some dyed fluid to flow through Banks Strait. The remaining majority then flows northward and eastward, again partly guided by the depth contours, as shown in Figs $3 b$ and 4.

In every case where $\Delta_{\rho}>0$ and the inflowing fluid flowed down the slope into the Tasman Sea region, to a depth dependent on $N$, the fluid subsequently moved northwards. However, no net northwards movement of the water column as a whole or net circulation within the Tasman Sea was observed in any of these experiments. Hence the northward motion of the dyed fluid is attributed to the local pressure gradient generated by the continuous supply. This suggests that the northward motion of Bass Strait water in the real Tasman Sea is locally generated and not due to a mean circulation within the Tasman Sea.

\section{Conclusions}

The locations of the fluid exiting from Bass Strait on the eastern side in winter as inferred by Tomczak (1985) and shown in Fig. 1 here, and notably the flux through Banks Strait, have been reproduced in a set of laboratory experiments. However, this is not consistent with Tomczak's conjectured motion within Bass Strait.

In the experimental model this flow pattern is guided by the depth contours within Bass Strait, which direct the flow toward the south-eastern corner. The resulting pressure causes flow through Banks Strait. Since the dynamical balances in the model are scaled to approximate those in the ocean, this suggests that the reason for this flow pattern in the ocean is the same. It is believed that this is the first demonstration that depth contours in shallow regions such as continental shelves may be able to control the flow pattern.

In the model, after the Bass Strait water flows down the slope into the Tasman Sea it flows northward along the slope, but there is no net motion or circulation in the Tasman Sea region as a whole. Hence the northward motion of the Bass Strait water is driven by its own pressure gradient, and we expect that the same process is equally valid in the ocean.

The consistency established here indicates that laboratory models of this type are useful for investigating some aspects of shallow shelf and sea flows.

\section{Acknowledgments}

Discussions with Humio Mitsudera were helpful in the course of this work, which was made possible by grants from the Australian Research Council and Shell Australia.

\section{References}

Baines, P. G., Hubbert, G. D., and Power, S. (1991). Fluid transport through Bass Strait. Continental Shelf Research 11, 269-93.

Mitsudera, H., and Baines, P. G. (1992). Downslope gravity currents in a continuously stratified environment: a model of the Bass Strait outflow. Proceedings, 11th Australian Fluid Mechanics Conference, Hobart, December 1992, 1017-20.

Tomczak, M. (1985). The Bass Strait water cascade during winter 1981. Continental Shelf Research 4, 255-78.

Manuscript received 3 August 1994; revised and accepted 7 October 1994 